\title{
Measuring Adverse Drug Events on Hospital Medicine Units with the Institute for Healthcare Improvement Trigger Tool: A Chart Review
}

\author{
Iris Lau and Allison Kirkwood
}

\begin{abstract}
Background: An adverse drug event (ADE) is a noxious, unintended response to a drug, occurring at doses used in humans for prophylaxis, diagnosis, or treatment of disease or for modification of physiological function. ADEs account for about one-quarter of all adverse events in Canadian hospitals. Canadian data on specific types of ADEs and commonly implicated drugs are lacking. In particular, there is a paucity of data on ADEs that occur during hospital admissions.

Objectives: The primary objective was to identify the incidence of ADEs in a sample of adult general medicine inpatients over a 1-year period. The secondary objective was to identify the 5 drugs most frequently responsible for ADEs in this setting.

Methods: A retrospective chart analysis was conducted for general medicine patients discharged from St Paul's Hospital in Vancouver, British Columbia, from January to December 2011. ADEs were identified using the Institute for Healthcare Improvement (IHI) Trigger Tool for Measuring Adverse Drug Events. The Naranjo criteria were applied to assess causality, and a physician independently authenticated the ADEs for preventability and harm using the categories of harm set out by the US National Coordinating Council for Medication Error Reporting and Prevention.

Results: Of the 204 patient encounters reviewed, 15 involved ADEs, which represented an incidence of $7 \%$ over the 1 -year study period. The 5 drugs most frequently implicated in ADEs were vancomycin, ciprofloxacin, ceftriaxone, piperacillin-tazobactam, and moxifloxacin.

Conclusions: The rate of ADEs during hospital admissions was substantial. These events may necessitate additional investigations and interventions and may prolong the hospital stay. The authors do not recommend the IHI Trigger Tool for Measuring Adverse Drug Events for efficient prospective detection of ADEs in manual chart reviews. Possible modifications to improve the utility of this tool might include incorporating it into a compatible electronic health record system with automated trigger detection.
\end{abstract}

Keywords: adverse drug events, adverse drug reactions, Institute for Healthcare Improvement, chart review

\section{Can J Hosp Pharm. 2014;67(6):423-8}

\begin{abstract}
RÉSUMÉ
Contexte : Un événement indésirable lié à un médicament (EIM) est une réaction nocive et non intentionnelle à un médicament qui survient lorsque que le médicament est utilisé selon les doses normales chez l'humain aux fins de la prévention, du diagnostic ou du traitement d'une maladie ou de la modification d'une fonction physiologique. Les EIM représentent environ le quart des événements indésirables dans les hôpitaux canadiens. Or, il n'y a pas assez de données canadiennes qui portent sur les catégories précises d'EIM et sur les médicaments qui y sont normalement associés. De plus, les données sur les EIM se produisant durant l'hospitalisation sont très rares.
\end{abstract}

Objectifs : L'objectif principal visait à identifier sur une période d'une année la fréquence des EIM dans un échantillon composé de patients adultes hospitalisés au service de médecine générale. L'objectif secondaire visait à découvrir quels étaient les cinq médicaments les plus souvent responsables d'EIM dans ce contexte.

Méthodes : Une analyse rétrospective des dossiers médicaux de patients ayant obtenu leur congé du service de médecine générale du St Paul's Hospital de Vancouver entre janvier et décembre 2011 a été menée. Le Trigger Tool for Measuring Adverse Drug Events (outil déclencheur permettant de détecter les EIM) de l'Institute for Healthcare Improvement (IHI) a servi à repérer les cas d'EIM. L'algorithme de Naranjo a été utilisé pour en évaluer la causalité. De plus, un médecin indépendant a validé les EIM quant à leurs caractères évitable et préjudiciable, et ce, à l'aide des catégories de préjudice du US National Coordinating Council for Medication Error Reporting and Prevention.

Résultats : Parmi les 204 hospitalisations évaluées, 15 présentaient des EIM, soit une fréquence de $7 \%$ au cours de la période d'étude d'une année. Les cinq médicaments le plus souvent en cause dans les événements indésirables étaient la vancomycine, la ciprofloxacine, la ceftriaxone, la pipéracilline-tazobactam et la moxifloxacine.

Conclusions : Le taux d'EIM durant les hospitalisations était important. Ces événements pourraient exiger des évaluations et des interventions supplémentaires et ils pourraient prolonger le séjour à l'hôpital. Les auteurs ne recommandent pas l'utilisation du Trigger Tool for Measuring Adverse Drug Events de l'IHI pour procéder à une détection prospective efficace des EIM lors d'une analyse manuelle des dossiers médicaux. L'un des moyens qui permettraient d'améliorer l'utilité de cet outil serait de 
l'inclure dans un système de dossiers de santé informatisés muni d'une fonction pour détecter automatiquement les éléments déclencheurs.

Mots clés : événements indésirables liés aux médicaments, effets indésirables des médicaments, Institute for Healthcare Improvement, analyse des dossiers

[Treduction par l'éditeur]

\section{INTRODUCTION}

Tn 2000, the US Institute of Medicine published its landmark Ireport on patient safety, To Err is Human, ${ }^{1}$ which spurred a culture of awareness and focus on patient safety. The report's authors estimated that as many as 98000 Americans died in hospital each year because of medical errors and noted that medication-related errors constituted one of the most common types of error. ${ }^{1}$

In 2004, Baker and others ${ }^{2}$ published the Canadian Adverse Events Study, the first study to describe the frequency and types of adverse events experienced by patients admitted to acute care hospitals in Canada. Their estimate of 7.5 adverse events per 100 admissions was drawn from data collected in 1 teaching hospital, 1 large community hospital, and 2 small community hospitals in each of 5 provinces (British Columbia, Alberta, Ontario, Quebec, and Nova Scotia). ${ }^{2}$ The data were categorized according to the procedures or events to which events were related, with 85 (23.6\%) of the 360 adverse events being related to drugs or fluids. The authors concluded that further research was needed to examine and characterize adverse events.

Earlier the same year, Forster and others ${ }^{3,4}$ published 2 studies on adverse events that examined transition points in a patient's trajectory of hospital care. The first of these studies described adverse events in patients discharged from the general internal medicine service at The Ottawa Hospital to home or to a seniors' residence. ${ }^{3}$ The second study defined the characteristics and timing of adverse events affecting inpatients who were receiving acute care at The Ottawa Hospital. ${ }^{4}$ The authors also classified the adverse events and concluded that drugs were responsible for more than $50 \%$.

More recently, Sikdar and others ${ }^{5}$ examined adverse drug events (ADEs) occurring in adult patients and leading to emergency department visits. They found that $2.4 \%$ of patients presenting to the emergency department at 2 tertiary care hospitals in St. John's, Newfoundland and Labrador, had a confirmed or possible ADE. ${ }^{5}$ On the opposite side of the country, Samoy and others ${ }^{6}$ studied ADEs that resulted in admission to the internal medicine service of Vancouver General Hospital over a 12 -week period. They found that the frequency of drug-related hospital admission was $24.1 \% .^{6}$

There is a scarcity of Canadian literature describing ADEs that occur after patients have been admitted to hospital. The aim of the current project was to determine the 1-year incidence of ADEs in a general medicine population at St Paul's Hospital in Vancouver, British Columbia, using the Institute for Health Improvement (IHI) Trigger Tool for Measuring Adverse Drug Events. ${ }^{7}$ This tool, designed for use during retrospective review of charts, lists the triggers that the IHI has found to be the most useful clues that an ADE has occurred.

This study also aimed to define the 5 drugs most frequently implicated in ADEs. In addition, it was of interest to identify the triggers that most often led to identification of ADEs. It was hoped that knowledge gained about high-risk medications and situations would enable the pharmacy department to collaborate with other health care professionals and hospital managers to improve existing policies and procedures to prevent ADEs.

\section{METHODS}

This retrospective analysis involved adult patients 18 years of age or older who were discharged from medicine wards (on the 7th floor) of St Paul's Hospital between January 1 and December 31, 2011. The 7th floor was chosen for its population of patients representative of a general medicine population in a hospital setting. All patients with a total length of stay of at least $24 \mathrm{~h}$ were included. Patients admitted for the sole purpose of rehabilitation and those admitted to the psychiatric service on the 7 th floor were excluded, as the IHI tool was not validated for use in those populations. Ethics approval was granted by the University of British Columbia Clinical Research Ethics Board, the Providence Health Care Research Ethics Board, and the Fraser Health Research Ethics Board. No external funding was secured for this review.

Taking into account the constraints of a residency project, a target convenience sample of 204 patient encounters was selected to provide an equal distribution of 17 patient encounters per month over one calendar year. A patient encounter was defined as a single patient-specific dataset encompassing time from admission to the general medicine service to discharge in isolated instances in time. During 2011, the total number of encounters for general medicine patients on the 7 th floor was 2676, for an average of 223 per month (data obtained from hospital database; Sunrise Clinical Manager, version 5.7, Eclipsys Corporation). The sample of 204 patient encounters used in the study represented $7.6 \%$ of all medicine encounters on the 7 th 
floor in 2011. Preliminary data from chart reviews conducted with the IHI Global Trigger Tool ${ }^{8}$ showed that about $35 \%$ of the charts contained evidence of an adverse event (C Ciarniello, Director of Risk Management, Patient Safety and Patient Relations, Providence Health Care; written personal communication, September 14, 2012). In the Canadian Adverse Events Study, $23.6 \%$ of the adverse events identified contained evidence that the event was related to a drug or fluid. ${ }^{2}$ St Paul's Hospital had characteristics similar to those of the hospitals included in the Canadian Adverse Events Study, and it was therefore assumed that roughly $8.3 \%$ of charts reviewed would yield an ADE. Review of 200 charts was thus expected to reveal about 17 ADEs. A computer-generated random list of patient encounters meeting the above criteria and equally distributed across months was created by the Health Records Department at St Paul's Hospital.

Two pharmacists (I.L., A.K.) independently reviewed each patient encounter using the IHI Trigger Tool for Measuring Adverse Drug Events, allotting a maximum time of 20 min per chart, as recommended by the IHI. ${ }^{9}$ Additional triggers and specific definitions of the template triggers provided were added to the trigger tool template, as suggested by the IHI. These predetermined triggers (see Table 1) prompted the reviewers to look throughout the chart for indications that an ADE had occurred. All triggers identified during the chart review were documented, regardless of whether they led to discovery of an

\section{Table 1. Description of Triggers Used in a Study of General Medicine Inpatients at St Paul's Hospital, Vancouver}

\begin{tabular}{ll} 
Trigger No. & \multicolumn{1}{c}{ Description } \\
\hline T1 & Diphenhydramine \\
T2 & Vitamin K \\
T3 & Flumazenil \\
T4 & Antiemetics \\
T5 & Naloxone \\
T6 & Anti-diarrheals \\
T7 & Sodium polystyrene \\
T8 & Glucose $<4$ mmol/L \\
T9 & Clostridium difficile-positive stool \\
T10 & Partial thromboplastin time $>100 \mathrm{~s}$ \\
T11 & International normalized ratio $>6$ \\
T12 & Leukocyte count $<3 \times 10 \% / L$ \\
T13 & Platelet count $<50$ 000/L \\
T14 & Digoxin $>2$ ng/mL \\
T15 & Rising serum creatinine $>30 \%$ of baseline \\
T16 & Oversedation, lethargy, falls \\
T17 & Rash \\
T18 & Abrupt cessation of medication \\
T19 & Transfer to higher level of care \\
T20 & Caution sheet updated \\
T21 & Other (e.g., discharge summaries, consults) \\
\hline
\end{tabular}

ADE. All suspected ADEs were assessed for causality using the Naranjo criteria, ${ }^{10}$ a set of 10 questions with potential answers of "yes", "no", or "unsure". The response for each question was converted to a numeric score, and scores for the 10 questions were summed to categorize the event as a possible, probable, or definite ADE. A physician authenticated the ADEs and assigned preventability and harm according to types of medication errors, as specified by the US National Coordinating Council for Medication Error Reporting and Prevention (NCC MERP). ${ }^{11}$ This set of 9 categories denotes different degrees of harm associated with ADEs.

All data were recorded within a standardized electronic spreadsheet, which was subsequently used to generate descriptive statistics. QuickCalcs (GraphPad Software Inc, La Jolla, California) was used to calculate the kappa score for extent of agreement between the 2 pharmacist reviewers. Kappa scores range from 0 to 1 , with higher scores indicating greater agreement between reviewers.

\section{RESULTS}

A total of 204 patient encounters that met the inclusion criteria were reviewed. The general characteristics of the study population were as follows: average age 81 years, approximately evenly split between the sexes (100 [49\%] men and 104 [51\%] women), and average length of stay 11 days. A total of $15 \mathrm{ADEs}$ were identified, representing an incidence of $7 \%$ over the 1 -year study period. The triggers most commonly leading to identification of an ADE were T20 ("caution sheet updated"; $n=12$ ADEs), T9 ("Clostridium difficile-positive stool"; $n=8 \mathrm{ADEs}$ ), and T21 ("other", e.g., discharge summaries, consults; $n=3$ ADEs) (Figure 1). The 5 drugs most frequently responsible for ADEs were vancomycin, ciprofloxacin, ceftriaxone and piperacillin-tazobactam (tie), and moxifloxacin (Figure 2). Assignment of Naranjo scores led to categorization of these events as "possible" or "probable" ADEs. The kappa score for interobserver agreement between the 2 pharmacist reviewers in determining the Naranjo scores was 0.21 .

The ADEs identified encompassed a range of events, including C. difficile-associated diarrhea, rash, vomiting, and neutropenia. Most of the ADEs (13/15) were classified as NCC MERP Class $\mathrm{E}$ or $\mathrm{F}$, defined as events that cause temporary harm resulting in intervention and/or prolonged hospital stay.

\section{DISCUSSION}

This retrospective study identified a 7\% rate of ADEs in a population of general medicine patients in an urban tertiary care hospital. ADEs were most commonly related to antibiotic therapy.

Although the focus of this study was on identifying ADEs that occurred during hospital admission, both pharmacist reviewers noted a number of ADEs that occurred before admission, most 


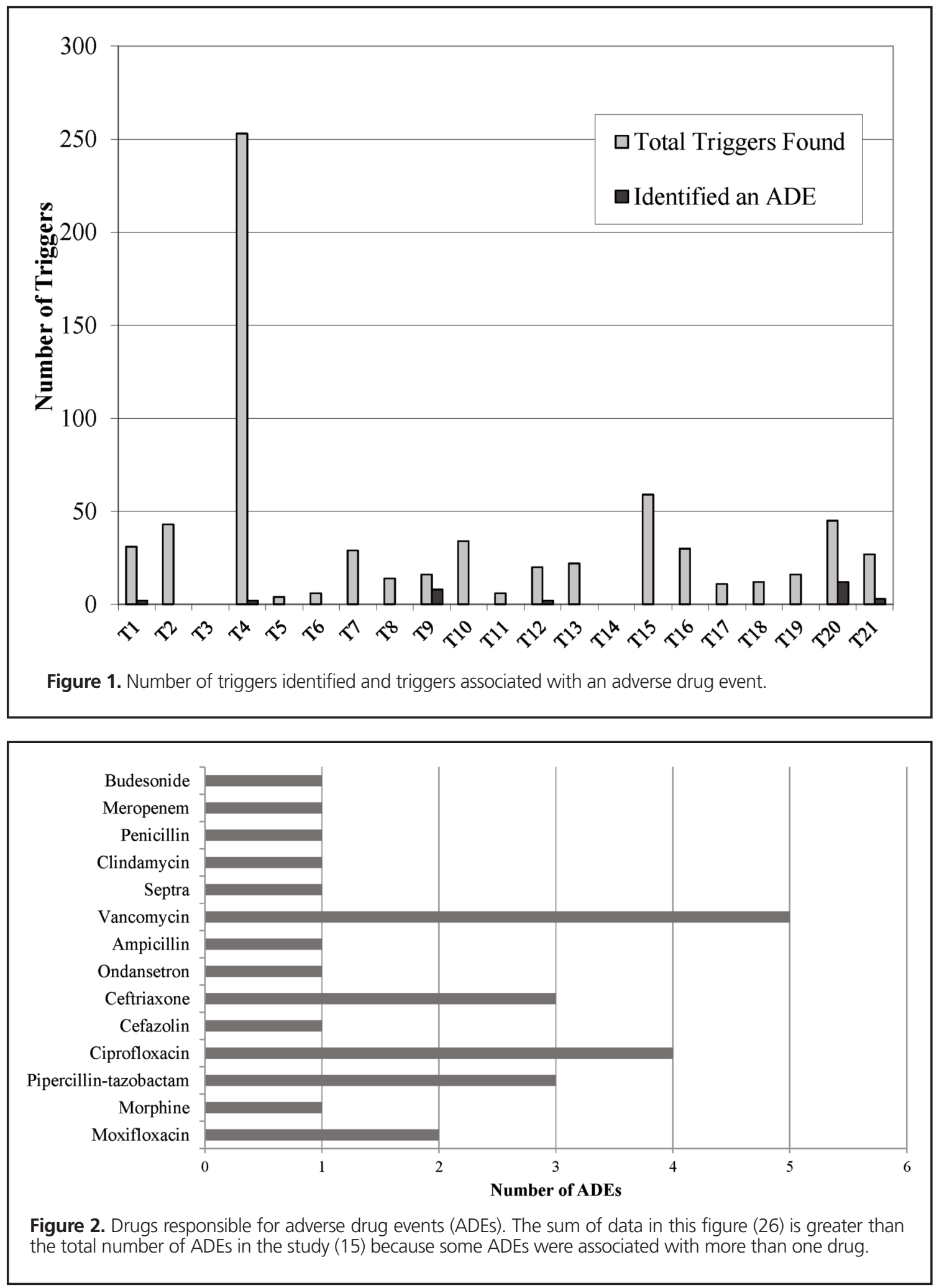

of which caused or contributed to the need for admission. These additional $13 \mathrm{ADE}$ were excluded from the analysis because they did not meet the a priori criterion of occurrence during the patient's hospital stay. This finding of pre-admission ADEs was consistent with previously reported Canadian data on ADEs leading to emergency visits and/or hospital admissions. ${ }^{5,6}$

There were striking differences between the ADEs captured in this study and events reported to the Patient Safety Learning 
System at the study hospital. Voluntary reports to the Patient Safety Learning System generally documented preventable errors occurring at the time of medication administration, such as medications or doses that were incorrectly administered and doses that were delayed or omitted (Kirkwood A, Wu H, Ng J. Patient Safety Learning System [PSLS] review findings. Presentation to Medicine Committee of St Paul's Hospital; April 2013). In the 204 patient charts reviewed for the current study, neither reviewer found any documentation of medication errors in the interdisciplinary progress notes or other permanent parts of the patient record. In contrast, ADEs captured using the IHI Trigger Tool for Measuring Adverse Drug Events were largely nonpreventable events related to inherent characteristics of the drug or its usage, only some of which were documented in the patient record.

Time and technical limitations prevented a thorough analysis of the drugs most frequently associated with ADEs (vancomycin, ciprofloxacin, ceftriaxone, piperacillin-tazobactam, and moxifloxacin) in relation to the drugs most commonly administered to general medicine patients on the 7 th floor. The computer system at St Paul's Hospital could provide data only for the medications most frequently ordered on this floor, specifically dalteparin, pantoprazole, acetylsalicylic acid, quetiapine, and prednisone (extract of orders entered between January 1 and December 31, 2011; Centricity Pharmacy Information System, version 8.2-198[33], General Electric Company). However, this computer-generated report did not take into account usage that would require manual tabulation of medications that were administered multiple times daily or confirmation of administration by review of the medication administration record. These potential aspects of the analysis were unfortunately beyond the scope of the current study but would be an interesting area for further research.

Some other limitations resulted from the methods and tools used in this study. In accordance with IHI recommended methodology, a maximum of 20 min was allotted to review each patient encounter. Although this time limit would streamline a quality assurance process, a period of $20 \mathrm{~min}$ is inadequate to reliably capture events that occurred during lengthy or complex admissions. Many of the triggers included in the IHI Trigger Tool for Measuring Adverse Drug Events were difficult to detect through manual review of the documents. For example, detection of trigger T16 ("oversedation, lethargy, falls") would necessitate the reviewer reading the entire interdisciplinary progress note and nursing assessment sections. Some triggers were found repeatedly but rarely led to identification of ADEs; for example, trigger T4 ("antiemetics") was identified 253 times, but only $1 \mathrm{ADE}$ related to this trigger was found. The time devoted to documenting triggers reduced the time available to review the patient record for ADEs.
Similarly, the Naranjo score for establishing causality contained parameters that were difficult to establish in a retrospective chart analysis. For example, definitive data on alternative causes or explanations for particular events could not be elicited by questioning care providers. It was also impossible to determine whether an ADE had occurred in response to a placebo, as well as the suspected drug. Scoring of causality was therefore influenced by the reviewer's perceptions of the documentation surrounding the event and inherent personal biases related to practice and life experiences. This subjectivity was reflected in the kappa score of 0.21 for interobserver agreement. Limited resources prevented having more than one physician authenticate the ADEs and designate their harm and preventability; as such, interobserver agreement for this phase of the study could not be quantified with a kappa statistic.

Finally, the a priori definition of ADEs did not include drugrelated events that did not cause harm or require an intervention or prolonged hospital stay, nor did it include events that occurred because a needed drug was omitted.

It was hoped that this study would establish the utility of the IHI Trigger Tool for Measuring Adverse Drug Events for retrospective quality assurance and perhaps encourage further research to explore the feasibility of using the trigger tool prospectively. However, given the limitations described above and the low correlation between identified triggers and ADEs, we would not recommend prospective manual use of the IHI Trigger Tool for Measuring Adverse Drug Events. The literature search conducted before development of the study protocol did not identify any manual tools or algorithms suitable for prospectively identifying ADEs during admission in a general hospital population. However, a future study is being considered to adapt the algorithm that Hohl and others ${ }^{12}$ used to identify communitybased ADEs in the emergency department, as a tool to identify ADEs in a general medicine population of inpatients.

For more effective retrospective application of the IHI Trigger Tool for Measuring Adverse Drug Events, it would be advisable to narrow the triggers according to common practices in targeted areas. For example, medications routinely used in order sets could be removed from the trigger list to improve efficiency of the tool. It would also be useful to set specific parameters or thresholds for triggers that describe increasing or decreasing trends. For example, the IHI tool suggests that a rise in serum creatinine over $30 \%$ above baseline should trigger additional review. Similarly, it may be beneficial to define new triggers, such as liver transaminase values more than 3 times the upper limit of normal, to capture ADEs that might have been missed in the current study.

We invite any practice sites with computerized patient health record systems to explore the possibility of adapting the IHI Trigger Tool for Measuring Adverse Drug Events for use as an electronic tool for prospective triage. A system with the ability to automatically identify patients who both have a trigger and 
are receiving medications that may lead to the trigger could assist clinicians in detecting ADEs.

The 7\% incidence of ADEs occurring over 1 year in the inpatient general medicine wards included in this study was not insignificant. These ADEs often resulted in additional interventions and may have prolonged patients' hospital stay. Further research is required to explore the use of trigger tools for detecting ADEs.

\section{References}

1. Kohn LT, Corrigan JM, Donaldson MS, editors. To err is human: building a safer health system. Washington (DC): National Academy Press; 2000.

2. Baker GR, Norton PG, Flintoft V, Blais R, Brown A, Cox J, et al. The Canadian Adverse Events Study: the incidence of adverse events among hospital patients in Canada. CMAJ. 2004;170(11):1678-86.

3. Forster AJ, Clark HD, Menard A, Dupuis N, Chernish R, Chandok N, et al. Adverse events among medical patients after discharge from hospital. CMAJ. 2004;170(3):345-9.

4. Forster AJ, Asmis TR, Clark HD, Al Saied G, Code CC, Caughey SC, et al. Ottawa Hospital Patient Safety Study: incidence and timing of adverse events in patients admitted to a Canadian teaching hospital. CMAJ. 2004;170(8):1235-40.

5. Sikdar KC, Alaghehbandan R, MacDonald D, Barrett B, Collins KD, Donnan J, et al. Adverse drug events in adult patients leading to emergency department visits. Ann Pharmacother. 2010;44(4):641-9.

6. Samoy LJ, Zed PJ, Wilbur K, Balen RM, Abu-Laban RB, Roberts M. Drugrelated hospitalizations in a tertiary care internal medicine service of a Canadian hospital: a prospective study. Pharmacotherapy. 2006;26(11):1578-86.

7. Trigger tool for measuring adverse drug events. Cambridge (MA): Institute for Healthcare Improvement; 2004 [updated 2011 Jun 7; cited 2012 Jul 28]. Available from: www.ihi.org/resources/Pages/Tools/TriggerToolfor MeasuringAdverseDrugEvents.aspx (login required to access content).

8. IHI Global Trigger Tool for measuring adverse events. Cambridge (MA): Institute for Healthcare Improvement; [revised 2008; cited 2014 Nov 27]. Available from: www.ihi.org/resources/Pages/Tools/IHIGlobalTriggerToolfor MeasuringAEs.aspx (login required to access content).
9. Griffin FA, Resar RK. IHI global trigger tool for measuring adverse events. 2nd ed. IHI Innovation Series white paper. Cambridge (MA): Institute for Healthcare Improvement; 2009. Available from: www.ihi.org/knowledge/ Pages/IHIWhitePapers/IHIGlobalTriggerToolWhitePaper.aspx

10. Naranjo CA, Busto U, Sellers EM, Sandor P, Ruiz I, Roberts EA, et al. A method of estimating the probability of adverse drug reactions. Clin Pharmacol Ther. 1981;30(2):239-45.

11. Types of medication errors. National Coordinating Council for Medication Error Reporting and Prevention; [updated 2001 Feb 20; cited 2012 Oct 3]. Available from: www.nccmerp.org/medErrorCatIndex.html

12. Hohl C, Bressler B, Wu S, Sheps S, Brubacher J, Dahri K, et al. Development of a screening strategy for community-based adverse drug related events in the emergency department. Edmonton (AB): Canadian Patient Safety Institute; 2010 [cited 2014 Nov 27]. Available from: www. patientsafetyinstitute.ca/English/research/cpsiResearchCompetitions/2007/ Documents/Hohl/Report/Hohl\%20Full\%20Report.pdf

Iris Lau, BSC(Pharm), ACPR, was, at the time of this study, a pharmacy resident with Lower Mainland Pharmacy Services, Vancouver, British Columbia.

Allison Kirkwood, BSC(Pharm), ACPR, MHA, is with the Pharmacy Department, St Paul's Hospital, Lower Mainland Pharmacy Services, Vancouver, British Columbia.

Competing interests: None declared.

Address correspondence to:

Iris Lau

10366 Hidden Valley Drive

Calgary AB T3A $4 Z 2$

e-mail: iolau@ualberta.ca

Acknowledgement

We would like to acknowledge Dr B Joan Gatto for authenticating the adverse drug events in terms of preventability and harm. We also thank Dr Michael Legal and Dr Stephen Shalansky for their input in the planning stages of this study.

\title{
CISADL 2014
}

\section{The 2014 Canadian Investigational \& Special Access Drug List is now on sale!}

\author{
Please place your order by completing our online form at \\ www.cshp.ca/productsServices/otherPublications/canadianInvestigational_e.asp
}

The CISADL is a compilation of drugs that are currently not marketed in Canada, and may have Investigational or Special Access status with Health Canada. This list is not endorsed by Health Canada's Special Access Program. For precise information about the regulatory status of a drug, contact the Special Access Program directly. Entries to the list are contributed to by several Drug Information Centers across Canada and by Canadian drug manufacturers. In cases where non-marketed drugs do not appear on the list, foreign references should be consulted. The content of the list includes generic name and strength/concentration, dosage form, pharmacology/therapeutic use, trade and code name, source (manufacturer/distributor), and comments. Available in English only. 